Revue de l'Institut des langues et cultures

d'Europe, Amérique, Afrique, Asie et Australie

$41 \mid 2020$

Escrituras nómadas en el mundo hispánico contemporáneo

\title{
Le Teatro Fronterizo de José Sanchis Sinisterra, ou comment nomadiser le drame
}

El Teatro Fronterizo de José Sanchis Sinisterra o cómo nomadizar el drama José Sanchis Sinisterra's Teatro Fronterizo or How to Nomadize the Drame

Laurent Gallardo

\section{OpenEdition}

Journals

Édition électronique

URL : http://journals.openedition.org/ilcea/11152

DOI : 10.4000/ilcea. 11152

ISSN : 2101-0609

Éditeur

UGA Éditions/Université Grenoble Alpes

Édition imprimée

ISBN : 978-2-37747-224-6

ISSN : 1639-6073

\section{Référence électronique}

Laurent Gallardo, «Le Teatro Fronterizo de José Sanchis Sinisterra, ou comment nomadiser le drame », ILCEA [En ligne], 41 | 2020, mis en ligne le 03 novembre 2020, consulté le 02 février 2021. URL : http:// journals.openedition.org/ilcea/11152; DOI : https://doi.org/10.4000/ilcea.11152

Ce document a été généré automatiquement le 2 février 2021.

(C) ILCEA 


\title{
Le Teatro Fronterizo de José Sanchis Sinisterra, ou comment nomadiser le drame
}

El Teatro Fronterizo de José Sanchis Sinisterra o cómo nomadizar el drama José Sanchis Sinisterra's Teatro Fronterizo or How to Nomadize the Drame

\author{
Laurent Gallardo
}

"Desde las zonas fronterizas no se perciben las fronteras"

José Sanchis Sinisterra,

El teatro fronterizo: manifiesto (latente)

1 La notion de nomadisme appliquée à la littérature conduit d'ordinaire à un questionnement que l'on pourrait formuler de la sorte: dans quelle mesure une écriture donnée est-elle le reflet du rapport que le nomade entretient au territoire ? On cherche ainsi à aborder des œuvres qui témoignent du nomadisme en tant que mode d'existence pour dégager les codes d'une représentation qui en serait le corollaire littéraire. Aussi pertinente que soit cette approche, nous souhaitons nous en détourner pour penser autrement le lien entre écriture et nomadisme. Notre réflexion s'inscrit, ce faisant, dans le sillage des travaux de Michel Collot (2014) sur "la géographie littéraire ", concept qui rend compte du tournant spatial que connaît de nos jours le discours critique. Si la modernité a longtemps privilégié la catégorie du temps au détriment de celle de l'espace, Michel Collot montre que la pensée postmoderne cesse d'envisager cette dernière comme un simple objet d'étude pour en faire un schème réflexif à part entière. Dans le domaine de la critique littéraire, ce changement de paradigme se traduit par l'émergence d'approches dites géocentrées, telles que la pensée-paysage (Collot, 2011), la géocritique (Westphal, 2007) ou encore la narratologie de l'espace (Ryan, 2003).

2 Une telle mutation s'inspire de l'importance croissante que revêt depuis un certain nombre d'années la spatialité dans le champ philosophique. En 1969, Michel Foucault suggérait déjà que notre époque "serait peut-être [celle] de l'espace» (2004: 12). La 
profusion des concepts topologiques chez Maurice Merleau-Ponty, Michel Foucault, Maurice Blanchot ou encore Jacques Derrida est à cet égard paradigmatique d'une redéfinition de la pensée sur des bases éminemment spatiales. Émerge ainsi, dans la seconde moitié du $\mathrm{xx}^{\mathrm{e}}$ siècle, une "géophilosophie » qui, selon Gilles Deleuze et Félix Guattari, repose sur l'idée que "penser n'est [pas] un fil tendu entre un sujet et un objet, ni une révolution de l'un autour de l'autre. Penser se fait plutôt dans le rapport du territoire et de la terre " (2005: 82). Conscients de la portée profonde d'une telle affinité entre les catégories du langage et de l'étendue, les deux philosophes cherchent à rompre avec une approche généalogique de la pensée afin d'aborder celle-ci dans sa dimension territoriale. Pour eux, "la géographie n'est pas seulement physique et humaine, mais mentale, comme le paysage. [...] Elle arrache [l'histoire] au culte des origines pour affirmer la puissance d'un milieu» (2005: 91). Cette reconfiguration épistémologique implique la création de nouveaux concepts (déterritorialisation, rhizome, ligne de fuite, nomadisme) liés à l'expérience spatiale. Dès lors, la philosophie cesse d'être une réflexion sur son histoire pour devenir une activité contingente qui met en jeu un certain rapport au territoire.

Poussant ce croisement avec la spatialité dans le champ littéraire, le poète francoécossais Kenneth White élabore, quant à lui, l'idée d'une géopoétique revendiquant la création comme géographie mentale. D'où la notion si prégnante chez White de « nomadisme intellectuel » (1987). À une pensée de type sédentaire, où chaque idée est vouée à un domaine, chaque signification à un objet, White oppose une pensée nomade, dérivante, qui refuse la segmentation de l'activité créatrice en champs clos et invite à penser l'errance comme condition de la création. Envisagé sous cet angle, le nomadisme ne renvoie plus nécessairement aux circonstances de l'écriture mais à une déterritorialisation qui lui est propre et qui, comme nous allons le voir, privilégie l'ouverture et la polytopie.

On ne peut, toutefois, saisir les enjeux de ce rapport à la création de manière globale. Jacques Ménétrier rappelle que le nomade se tient toujours en dehors des codes car il porte en lui «le refus de la domestication» (1972: 48). Comme l'ont montré Gilles Deleuze et Félix Guattari, il n'évolue pas au sein d'« une totalité englobante, mais se déploie au contraire dans un milieu sans horizon " (1980:469). Son espace est, de ce fait, un local absolu (un désert, une steppe, une mer) engendré par une série d'opérations singulières. Voilà pourquoi il nous faut envisager le nomadisme comme une activité contingente, inscrite dans une territorialité tangible, bien que non nécessairement matérielle. En abordant l'œuvre du dramaturge espagnol José Sanchis Sinisterra comme manifestation paradigmatique de ce "nomadisme intellectuel», ce sont précisément ces opérations et leur impact sur un territoire déterminé - le champ théâtral - que nous voulons explorer ici. Nous proposons, pour ce faire, de mener cette réflexion en posant d'abord la question de la territorialisation de l'œuvre telle que la conçoit le dramaturge et en explorant ensuite la pratique de l'adaptation théâtrale comme opération particulière de cette création nomadique. On interrogera enfin les enjeux d'une pensée du dehors appliquée à la création théâtrale : est-elle l'expression d'un théâtre postdramatique résolument interdisciplinaire ou bien revendique-t-elle le primat du drame pour en faire un terrain mouvant d'expérimentations incessantes? 


\section{Investir la frontière pour penser le théâtre en ses dehors}

5 L'œuvre de José Sanchis Sinisterra naît d'un désir de liberté qui l'engage en tant que créateur et qui, dans le même temps, ouvre le théâtre sur un devenir singulier. Dès ses débuts dans les années 1960, le dramaturge ne cesse de quitter le territoire étriqué d'un art sans profondeur, oscillant entre le pur divertissement et un élitisme culturel qui le détourne de son essence populaire. À l'en croire, ce n'est pas le théatre qui est en crise, mais une certaine théâtralité qui cherche à confondre le monde avec sa représentation pour faire de la scène le lieu d'un formatage. Ce langage aliénant, José Sanchis le nomme Système théâtral bourgeois ${ }^{1}$ et c'est en résistance à celui-ci que se forge sa réflexion dramaturgique. Tout comme Brecht, il perçoit qu'il ne peut y avoir de régénération du théâtre sans transformation esthétique, car c'est bien dans les codes de la théâtralité que l'idéologie s'immisce. Dès lors, son entreprise s'apparente à une véritable déconstruction de ce système pour faire advenir un art, non plus du reflet, mais du questionnement, portant sur la réalité un regard distancié et critique. Dans le prologue de Sangre lunar, le dramaturge s'interroge sur le sens d'une telle démarche :

¿Por qué esa obcecada voluntad de proceder a la deconstrucción de la estructura dramatúrgica convencional? ¿A qué se debe la casi ciega obediencia a impulsos, si no del todo irracionales, sí al menos alógicos, desasidos de las coordenadas que sustentan nuestra percepción de la realidad? ¿Acaso me habitaba la sospecha -que he expresado a menudo irónicamente- de que la realidad no es realista? $(2010,77)$

6 C'est précisément cette recherche d'une représentation de la réalité en marge du réalisme qui le pousse à ouvrir sa réflexion sur d'autres conceptions théâtrales. On pourrait citer, à titre d'exemples, la récupération des genres mineurs de la scène baroque espagnole dans Ñaque o de piojos y actores (2001) ou encore celle des formes brèves du théâtre beckettien dans Pervertimento $y$ otros gestos para nada (1997). Il s'intéresse également à des disciplines scientifiques (la physique quantique, la théorie du chaos ou encore la cybernétique) qui interrogent les conceptions admises du temps, de l'espace et de l'observation. Ces trois notions sont généralement traitées au théâtre comme des réalités immanentes de sorte que la supposée unité de l'espace-temps marque un seuil au-delà duquel la représentation théatrale ose rarement s'aventurer. Or, depuis plus d'un demi-siècle, la physique quantique n'a eu cesse de redéfinir les conditions d'existence de la réalité en marge de la pensée newtonienne. En développant la théorie des mondes multiples, Hugh Everett a notamment émis l'hypothèse que notre réalité coexiste avec de nombreux autres mondes inaccessibles entre eux. Dans Perdida en los Apalaches (juguete cuántico) [1991], José Sanchis a recours à cette théorie pour développer une fiction théâtrale qui, en intégrant l'idée d'univers multiples, rompt avec le réalisme. Si la pièce débute par une conférence du professeur Dorothy Greñuela sur les distorsions de l'espace-temps, à mesure que l'action progresse, d'autres univers se matérialisent sur scène. Il y a d'abord le personnage de l'Intrus qui transporte les spectateurs dans les années 1960. Puis, c'est dans le futur que voyage Dorothy Greñuela, persuadée que le professeur Forrester, chercheur en physique quantique, est en train de lui jouer un mauvais tour. Les différents univers finissent par se rejoindre et s'entremêler au sein d'une représentation qui intègre les distorsions spatiotemporelles. On assiste ainsi à une déconstruction du « bel animal » aristotélicien par le biais d'un rapprochement avec la physique quantique. En d'autres termes, il s'agit, pour 
le dramaturge, de faire avec le théâtre ce que cette nouvelle science fait avec la physique classique.

7 La frontière, en tant qu'espace symbolique, devient le lieu privilégié d'une ouverture pour penser le théâtre hors de lui-même. Elle permet de multiplier les rencontres avec des champs artistiques et scientifiques qui alimentent une recherche proprement théâtrale. Ces rencontres participent d'un nomadisme intellectuel, par lequel José Sanchis cherche moins à fuir le théâtre qu'à le faire fuir. Ce qui compte, c'est le rapport d'usage qu'il instaure avec ses différents intercesseurs afin de les mettre au service d'une expérimentation formelle. En perpétuelle évolution, son théâtre prend ainsi la forme d'un territoire mouvant aux limites incertaines et changeantes qui en 1977 cristallisera dans la création du Teatro Fronterizo.

8 À mi-chemin entre la troupe et le groupe de recherche, ce collectif se veut un lieu de rencontres pour des individus venus d'horizons divers. C'est en ces termes que José Sanchis le définit :

El Teatro Fronterizo es un grupo abierto. Abierto a prácticas de expresión y comunicación no teatrales e incluso no artísticas, por considerar que la teatralidad no es algo definitivamente establecido por los sistemas y códigos tradicionales, sino por una dimensión humana de reconocimiento y autoconstrucción que cambia con el hombre, que precede, acompaña o sigue sus deseos de cambio. (2002: 37$)$

9 L'accent est mis sur le rejet du drame conventionnel et la nécessité de susciter une alternative s'inspirant de champs de création a priori éloignés de la pratique scénique contemporaine. Précisément, ce désir d'explorer des formes nouvelles explique pourquoi il est si difficile d'assigner à l'œuvre de José Sanchis une place fixe dans la cartographie réglée du drame contemporain. Ignorant les lignes de segmentation classiques, elle échappe à toute tentative de sédentarisation forcée en un cadre préétabli. La situer dans le voisinage exclusif de Brecht revient à négliger les rapports d'affinité qui la lient à l'œuvre de Beckett; l'analyser dans le contexte unique du théâtre espagnol, c'est omettre l'influence déterminante de cette œuvre sur les dramaturges catalans des années $1980^{2}$. La même difficulté réapparaît lorsqu'il s'agit de circonscrire son activité en un champ particulier : est-il un dramaturge qui s'adonne à la mise en scène? Un théoricien qui écrit? Un enseignant qui théorise? L'intéressé reconnait lui-même que sa situation dans le théâtre est absolument frontalière :

Soy un autor porque elaboro mis propios textos, pero no soy un autor convencional; soy un director, pero no del todo porque hago sólo los montajes de mis textos; y además soy profesor de teatro, un investigador, un teórico... Este es uno de mis problemas, mi falta de ubicación en el panorama teatral. (Boadella, Onetti, Sanchis \& Solano, 1999 : 110)

Mais n'est-ce pas le propre du créateur, pris au sens fort, que de dépasser les dualismes traditionnels pour ouvrir son art à des pratiques et des formes d'expression nouvelles? Ne cherche-t-il pas toujours, souvent sans y parvenir tant la tâche s'avère difficile, à déborder le réel pour conquérir, en l'objectivant, l'espace de l'informe? En vérité, ce n'est pas le manque de localisation de l'œuvre qui fait problème, mais la grille de lecture qu'on lui applique et qui préside à la segmentation de l'activité créatrice en champs clos de sorte que tout ce qui n'entre pas dans la grille ne peut être conçu ni perçu. Or, le théâtre de José Sanchis fonctionne sur le mode inverse : le processus de création est indissociable d'un mouvement de déterritorialisation qui consiste à abandonner « los locales inequívocos, los recintos consagrados, los comportamientos netamente serviles a sus rótulos, las designaciones firmemente definidas por el consenso colectivo o 
privativo » (Sanchis, 2002: 36). Le dramaturge défend d'ailleurs l'idée que son rapport au théâtre est intrinsèquement marqué par la contingence :

Todo lo que pueda decir son opiniones de una subjetividad muy peculiar, desde una determinada trayectoria y desde una posición siempre inestable, siempre en movimiento, $y$ en la que procuro no quedarme anclado en las certezas. [...] Tengo siempre una actitud muy dubitativa y relativista. (1999: 110)

11 La question n'est donc pas tant de savoir à quelles déterminations génériques ou bien esthétiques l'œuvre se rapporte, mais comment elle parvient à se soustraire aux grands ensembles pour faire advenir une géographie inédite, pour se reterritorialiser ailleurs, en un espace nouveau où le théâtre tend tout entier vers son dehors.

Cet ailleurs, horizon idéal d'une création in progress, prend la forme d'une frontière, concept majeur de la poétique de José Sanchis qui condense de façon indissoluble des éléments géographiques, politiques et dramaturgiques. Le point de départ pour une approche de la frontière pourrait être le texte-programme, «El Teatro Fronterizo: Manifiesto (latente) ", où le dramaturge expose sur un mode à la fois poétique et théorique ses propres conceptions dramaturgiques. Il s'efforce ainsi de penser la présence d'une spatialité, d'une extension et d'une extériorité, des questions de limites et de territoires jusqu'à mettre en lumière une proximité certaine entre le registre de la géographie et celui du discours théorique.

Le texte s'ouvre sur la description d'un territoire frontalier : « Hay territorios en la vida que no gozan del privilegio de la centralidad. Zonas extremas, distantes, limítrofes con lo Otro, casi extranjeras" (2002: 33). Frontière qui n'est ni proprement référentielle, ni proprement métaphorique ; lieu sans identité précise, no man's land permissif, ouvert sur l'extérieur et qui s'oppose au "centre». Chez José Sanchis, la centralité est marquée par le privilège, confirmant ainsi ce qu'en disent les géographes ${ }^{3}$ qui définissent le centre comme un espace dominant en cela qu'il concentre en un lieu donné des instances de pouvoir et s'impose à une périphérie dont la marginalité s'accroît en fonction de son éloignement.

Mais l'opposition centre/frontière est sensiblement différente de l'opposition centre/ périphérie. Cette dernière implique un rapport dichotomique, le centre existant seulement comme antithèse de la périphérie. Or, le couple centre/frontière met en jeu un espace inédit, celui de l'extériorité. Cette nouvelle distribution du territoire diffère de la première car elle vise à subsumer un rapport binaire qui méconnaît la multiplicité des différences en les réduisant au principe unique de la contradiction. La frontière, telle que la conçoit José Sanchis, prend précisément la forme d'un lieu de rencontres, d'un espace de compromissions, où sont possibles «los lindes transitables entre dominios en apariencia distantes, los encuentros entre dos campos que se ignoraban mutuamente » (Sanchis, 2002 : 35). Investir la frontière revient en somme à refuser les systèmes aprioristiques, les arborescences prédéterminées pour libérer la création d'une logique dualiste. "Todas las dicotomías son falsas", souligne José Sanchis, «[t]enemos que liberarnos del pensamiento dicotómico parar poder avanzar en un sentido creativo » $(2009: 13)$.

Dans la deuxième partie du manifeste, la frontière accède à la catégorie d'espace mental: « Hay gentes radicalmente fronterizas. Habiten donde habiten, su paisaje interior se abre siempre sobre un horizonte foráneo » (2002 : 24). Le dramaturge insiste ici sur l'activité nomade de ces individus frontaliers: "Viven en un perpetuo vaivén que ningún sedentarismo ocasional mitiga » (2002: 34). Il s'agit d'un mouvement intérieur, d'une 
migration permettant d'accéder à un espace au-delà des enclos de la finitude: "[Cuando] el paisaje comienza a poblarse y a delimitarse, [...] parten hacia adentro o hacia afuera, hacia un lugar sin nombres conocidos" (Sanchis, 2002 : 34). Cette poétique de la frontière est de ce fait indissociable d'une revendication du mouvement, de l'inachèvement, de la création comme quête d'un ailleurs.

Une telle approche donne alors lieu à une culture frontalière, qui est le thème de la troisième partie du manifeste: "Hay una cultura fronteriza también, [...] una cultura centrífuga, aspirante a la marginalidad, aunque no a la marginación -que es a veces su consecuencia indeseable-, y a la exploración de los límites, de los fecundos límites » (Sanchis, 2002 : 35). José Sanchis définit cette culture frontalière comme un champ de résistance face aux productions dominantes. Elle suppose à la fois un éloignement physique et intellectuel vis-à-vis des instances de pouvoir et une remise en cause des classifications académiques dans le but d'instaurer des rapports d'échange avec d'autres formes de création, au sein d'un univers sensible aussi large que possible et dont la carte est toujours à reprendre. Le théâtre de José Sanchis est en cela très proche du mouvement géopoétique initié par Kenneth White, qui explore dans son œuvre les chemins d'un rapport à l'espace terrestre fluide et jamais figé ; rapport indissociable, comme le souligne White, d'une volonté de rupture :

[Il s'agit] de briser un espace circonscrit par les idéologies, les croyances, les politiques culturelles nationales, afin d'ouvrir l'esprit de chacun aux mouvements, aux métamorphoses du monde, de ce monde qui est pour l'homme ce qui est de plus proche et ce dont il est le plus séparé. (1987: 45)

Le dramaturge cherche, en d'autres termes, à expérimenter une nouvelle façon d'occuper le champ théâtral, éloignée des formes de création sédentaires, ancrées dans des axiomatiques closes. Il vise ainsi à instaurer un nouveau rapport à la création en accord avec une forme de nomadisme intellectuel. "Una de [mis] metas más precisas", déclare-t-il dans le manifeste, « sería suscitar la emergencia de pequeñas patrias nómadas, de efímeros países donde el pensamiento y la acción hubieran de inventarse cada día » (2002:36). Gilles Deleuze et Félix Guattari ont montré que le nomade se définit moins par une mobilité permanente que par le fait de se distribuer en un espace lisse : «Il occupe, il habite, il tient cet espace, et c'est là son principe territorial» $(1980: 444)$. Cette territorialité exclusivement nomade constitue une forme de résistance face à l'appareil d'État qui se caractérise par sa capacité à «strier l'espace sur lequel il règne [...], à capturer des flux de toutes sortes, de population, de marchandises ou de commerce, d'argent ou de capitaux » $(1980: 445)$. Ainsi, le nomade est-il celui qui, en instaurant un espace lisse là où règne le quadrillage du pouvoir, crée une zone de liberté. Les deux philosophes font à cet égard l'analyse suivante :

Chaque fois qu'il y a une opération contre l'État, indiscipline, émeute, guérilla ou révolution comme acte, on dirait qu'une machine de guerre ressuscite, qu'un nouveau potentiel nomadique apparait, avec reconstitution d'un espace lisse. C'est en ce sens que la réplique de l'État, c'est de strier l'espace contre tout ce qui risque de le déborder. (1980:246)

18 La frontière telle que la conçoit José Sanchis s'apparente à cet espace lisse du nomade, elle est le lieu d'une expérimentation théâtrale que le système théâtral bourgeois ne parvient pas à coloniser et qui outrepasse les lignes de segmentation classiques dans le but de susciter des rencontres inespérées entre des êtres et des champs de création apparemment sans rapport. Dans le manifeste du Teatro Fronterizo, le dramaturge rappelle que son objectif est bien de «provoca[r] inesperadas conjunciones o delatar la 
estupidez de viejos cismas, pero también destruir los conjuntos armónicos, desarticular venerables síntesis y hacer, de tan sola de una de sus partes, el recurso total de sus maquinaciones » (2002: 36). On le voit, la frontière est donc moins une métaphore du théâtre de José Sanchis qu'un concept poétique, véritable foyer de création, central par la condensation qu'il opère et relatif en cela qu'il interagit avec d'autres concepts, comme la marginalité, l'entre-deux ou encore le translucide ${ }^{4}$.

Il existe enfin, au sein même de cette culture frontalière, un théâtre frontalier, hybride et expérimental qui s'oppose au théâtre établi, "esa institución que languidece en la periferia de nuestra vida social, a pesar de que sus salas se levantan en el centro de la Ciudad» (Sanchis, 2002: 37). Du centre vers la frontière, ce que propose le manifeste n'est pas un exil forcé vers des contrées lointaines, plus accueillantes, sorte de renoncement au théâtre. Cette activité déterritorialisante est à la fois un mouvement que José Sanchis fait et qui défait la théâtralité établie en l'ouvrant sur d'autres langages. C'est en somme l'expérimentation des infinies possibilités du théâtre qui l'intéresse, recherche ayant pour but de faire apparaitre la contingence des conventions théâtrales et de créer ainsi les conditions d'une théâtralité alternative au Système théâtral bourgeois. Dans le prologue de la Escena sin límites, recueil des essais théoriques de José Sanchis, Juan Mayorga insiste sur cette dimension expérimentale :

Sanchis ha descubierto teatro alli donde casi nadie lo había siquiera intuido. Una y otra vez nos ha obligado a reconocer que teníamos del arte teatral una imagen incompleta, insuficiente. Ese inconformismo es el motor de su teatro fronterizo, que escoge el límite como su materia. Sometiéndola a una desestabilización permanente, Sanchis descubre que, del otro lado de la frontera, hay más teatro. (2002:25)

D'ailleurs, le dramaturge se définit lui-même comme "hacedor de teatro " (2002:42), expérimentateur engagé dans un mouvement qui vise non pas le champ théâtral tel qu'il est mais son dehors. Tout l'enjeu de l'œuvre réside dans la recherche de ce devenirautre du drame, difficilement repérable car il n'a rien à voir avec les systèmes d'organisation du drame actuel, ni avec son histoire officielle, mais passe plutôt par des œuvres que l'histoire du théâtre laisse généralement de côté.

Pratiquer ce théâtre frontalier revient donc à nomadiser la théâtralité en l'ouvrant sur ses dehors. L'œuvre prend alors la forme d'un territoire connecté avec d'autres dramaturgies (celles de Brecht et de Beckett), d'autres genres (le roman qui donnera lieu à de nombreuses adaptations théâtrales) et d'autres disciplines (la physique quantique, la théorie du chaos ou encore la cybernétique), permettant ainsi de déconstruire le drame pour mieux le transformer.

\section{La narraturgia : une pratique nomadique}

Précisément, l'adaptation théâtrale telle que la conçoit José Sanchis offre un exemple suggestif pour comprendre les enjeux de ce nomadisme intellectuel appliqué au champ théâtral. Les premières années du Teatro Fronterizo sont marquées par la pratique assidue de cette modalité de réécriture que le dramaturge préfère nommer narraturgia (2012), néologisme construit par la juxtaposition de narración et dramaturgia. Il définit d'ailleurs cette pratique comme "un territorio fronterizo e impuro en el que se entrelazan inextricablemente ambos géneros, el narrativo y el dramático " (2012: 17). Elle donnera également lieu à un essai, Narraturgia: dramaturgia de textos narrativos, où José Sanchis expose en des termes à la fois théoriques et pratiques ses conceptions de l'adaptation 
théâtrale. Au fil des années, il a ainsi transposé à la scène des auteurs tels que Franz Kafka, Herman Melville, James Joyce, Samuel Beckett, Julio Cortázar ou encore Ernesto Sábato. Le dramaturge se dit notamment intéressé par des textes « que, por una parte, no prefiguran una representación convencional $y$, por otra parte, se sitúan en zonas particularmente refractarias a la domesticación cultural burguesa: textos excéntricos [...] en relación con el discurso dominante » (2002:191).

De ce fait, la narraturgia apparaît d'emblée comme une modalité de réécriture permettant de confronter la théâtralité à des œuvres porteuses d'un renouveau formel. Il s'agit, comme le souligne le dramaturge, «de cuestionar desde el exterior la práctica habitual de la estructura dramática tratando de ensanchar las fronteras de la teatralidad» (2012: 20). Ainsi donc, cette pratique hypertextuelle ne vise pas seulement la projection d'une œuvre sur la scène car le dramaturge tend aussi, par le biais de ce transfert, à provoquer un dérèglement des conventions théâtrales. En ce sens, la narraturgia ne doit pas être perçue comme une fin en soi, mais comme un moyen dramaturgique au service d'un processus expérimental portant sur les paramètres de la théâtralité établie.

D'où la nécessité de postuler une conception élargie de cette modalité de réécriture afin de prendre en compte non seulement le processus de transformation de l'œuvre originale en œuvre adaptée, mais aussi ses incidences sur le cadre d'accueil. Dans son étude de l'adaptation cinématographique, André Helbo établit un parallélisme suggestif entre cette pratique et une opération biologique d'altération que les généticiens nomment transduction :

Le terme transduction inventé par Lederberg, prix Nobel de médecine en 1958, désigne la transmission de matériel génétique d'une bactérie à une autre par l'intermédiaire d'un troisième organisme colonisateur; le mécanisme de contamination suppose la transmission partielle de l'héritage génétique de l'organisme bactériophage mais aussi celui de la bactérie phagocytée initialement par cet organisme. (2006:21)

Pour André Helbo, l'adaptation ne peut être envisagée comme un simple transfert linéaire et unidirectionnel car, tout comme la transduction, elle instaure un " processus de réévaluation réciproque lié aux instances médiantes servant à intérioriser des contraintes contextuelles et institutionnelles pesant sur l'énonciation » $(2006: 22)$. En d'autres termes, une telle pratique doit nécessairement donner lieu à ce que le sémioticien nomme une approche polysystémique, « définissant le réseau complexe de relations entre les systèmes culturels, la trajectoire, les opérateurs et la nature du transfert " (Helbo, 2006: 22). Il s'agit de dépasser le stade de l'analyse comparative entre texte-source et texte-cible pour envisager l'adaptation dans une dimension sémiotique globale afin d'appréhender l'impact du transfert adaptatif sur le cadre énonciatif d'arrivée.

Pour en revenir au théâtre, on pourrait affirmer que l'adaptation, en tant que transformation d'un hypotexte narratif en hypertexte théâtral, implique l'élaboration d'une dramaturgie inédite capable d'intégrer des contraintes extra-théâtrales. On pourrait d'ailleurs établir une distinction entre l'adaptation-domestication qui constitue une simple transmodalisation - où il s'agit d'adapter une œuvre aux conventions dramatiques en vigueur- et l'adaptation-subversion qui tend à s'affranchir des codes de la théâtralité, en confrontant ceux-ci à un dispositif discursif extrinsèque, celui de l'hypotexte. Dans ce dernier cas, c'est le cadre conventionnel d'accueil qui s'adapte aux spécificités de l'œuvre et non l'inverse. 
27 Prenons à titre d'exemple La noche de Molly Bloom de José Sanchis, adaptation du long monologue intérieur qui clôt le dernier chapitre d'Ulysse de James Joyce. Dans le prologue de la pièce, le dramaturge insiste sur la nature éminemment subversive de son travail adaptatif :

Esta tarea constituye a todas luces, una enorme traición, resultado de la suma -y algo másde una serie de pequeñas infidelidades. [...] Traicionar la textualidad de Joyce, pues, para instaurar la teatralidad; pero también asumir la textualidad de Joyce para traicionar la teatralidad, para crear las condiciones de una discreta subversión escénica. (2002:60)

La narraturgia telle que la pratique le dramaturge s'apparente, on le voit, à une opération qui ne cherche pas à établir un lien de fidélité avec l'hypotexte, mais plutôt un rapport de subversion créative, qui affecte également la théâtralité puisqu'il s'agit de confronter celle-ci à l'écriture de Joyce pour faire advenir une forme théâtrale inédite.

De toutes les adaptations de José Sanchis, La noche de Molly Bloom est peut-être celle où la transformation de l'hypotexte est la plus légère, le dramaturge se limitant à sélectionner dans le texte de Joyce une série de fragments afin de composer un portrait fragmentaire de Molly Bloom. Alors que cette dernière ne parvient pas à trouver le sommeil, elle laisse libre cours à ses pensées les plus intimes et passe en revue sa journée, marquée par la rencontre avec son amant, puis celle de son mari, Leopold Bloom, qu'elle soupçonne d'être infidèle. Les souvenirs de son enfance et des relations extraconjugales qui ont jalonné son existence laissent place à des réflexions désordonnées sur le désir, la vie familiale et les multiples tracas du quotidien. L'originalité de ce monologue réside essentiellement dans sa syntaxe, le texte se divisant en huit paragraphes composés chacun d'une seule phrase et privés de ponctuation. Ce flux de paroles chaotique donne accès à une pensée libre qui se déploie par associations d'idées et semble répondre à des mouvements irrationnels. Affranchies des convenances stylistiques et morales, les pensées de Molly se présentent sans pudeur et sans ornement. Comme le souligne José Sanchis, dans ce flux de parole ininterrompu, « la frustración, el desvalimiento, la resignación insatisfecha ante una realidad humana desencantada y agobiante, se entrelazan con el vitalismo, la sensualidad y la libertad interior de esta mujer sin atributos, a la vez individuo concreto y representación de la ciega energía de la naturaleza » (2002: 59).

Paradoxalement, cette profusion verbale donne lieu, dans l'adaptation de José Sanchis, à un dispositif théâtral des plus sommaires :

En el centro del escenario, primer término, se configuran un fragmento de dormitorio: jirones de realidad doméstica emergiendo del ámbito oscuro y vacío. A ambos lados, plenamente visibles, dos focos con sus pies enmarcan el área de ficción, delimitada en su base por una gran alfombra. (Sanchis, $1996: 13$ )

31 De ce décor minimaliste, menacé par le vide et l'obscurité, émerge alors la silhouette de Leopold Bloom endormi aux côtés de Molly. Puis, c'est le visage et le corps de celle-ci que la lumière éclaire avant que ne débute son long monologue. Le couple apparaît comme un avatar du duo formé par Willie et Winnie dans Oh les beaux jours de Samuel Beckett. On retrouve ainsi dans la pièce cette tension proprement beckettienne entre une parole foisonnante et une action dramatique précaire et stagnante. D'un point de vue formel, l'adaptation permet le transfert dans le champ théâtral du monologue intérieur tel que le conçoit et le pratique James Joyce. Édouard Dujardin a notamment montré que cette forme littéraire «a pour objet d'évoquer le flux 
ininterrompu des pensées qui traversent l'âme du personnage au fur et à mesure qu'elles naissent sans en expliquer l'enchaînement logique» (1931: 237). En se présentant tel un discours de l'inconscient, il déjoue tout déploiement narratif linéaire jusqu'à apparaître comme antérieur «à toute organisation logique [...] de façon à donner l'impression d'un tout-venant " (Dujardin, 1931: 237). Le monologue intérieur permet ainsi à James Joyce d'échapper au récit classique pour développer une structure discursive non-linéaire. C'est d'ailleurs cette spécificité du monologue intérieur qui incite Gérard Genette à le rebaptiser " discours immédiat » car, selon lui, « l'essentiel, comme il n'a pas échappé à Joyce, n'est pas qu'il soit intérieur mais qu'il soit d'emblée (dès les premières lignes) émancipé de tout patronage narratif, qu'il occupe d'entrée de jeu le devant de la scène » $(1972: 193)$.

C'est en des termes semblables que José Sanchis définit la visée dramaturgique de son adaptation :

La noche de Molly Bloom renuncia a la trama, al argumento, a la intriga, a la fábula, en suma, que es considerada, desde Aristóteles hasta Brecht, la columna vertebral de toda acción dramática. La propia acción es sustituida por una sucesión de acciones, de actos, de gestos, cuyo dinamismo no viene determinado por el clásico encadenamiento de deseos $y$ obstáculos, por la dialéctica del conflicto. (2002:60)

Ainsi le monologue intérieur, en tant que forme libre, fait émerger une dynamique théâtrale alternative où le conflit intérieur du personnage, qui naît de la tension entre la conscience et l'inconscient, donne lieu à une dramaturgie de la parole permettant à l'action dramatique de s'émanciper de l'intrigue. Libérée du récit classique, la parole théâtrale se propage suivant une dynamique discursive dont la logique interne s'apparente à une pensée chaotique mais non moins productrice de sens. En adaptant James Joyce, José Sanchis tend donc à une mise à distance des conventions théâtrales au profit de " una deconstrucción de las pautas de la teatralidad que podríamos llamar inmanente, de la teatralidad implícita, de la teatralidad que inevitablemente nos habita» (Sanchis, 2012 : 17). Il reprend ainsi à son compte cette ligne de fuite que l'œuvre de Joyce opère dans le roman moderne en s'affranchissant des normes du récit. Le lien de fidélité, propre à toute adaptation, réapparaît alors dans l'attention que le dramaturge porte à ce mouvement de subversion créative.

Tandis que l'adaptation classique instaure une domestication de l'œuvre originelle en l'ajustant à un contexte théâtral donné, José Sanchis choisit de situer la narraturgia dans une perspective opposée, qui tend non pas à une normalisation de l'œuvre adaptée, mais au contraire à une déstabilisation de la théâtralité établie. Cette pratique est autant écriture que réécriture en cela qu'elle suppose une expérimentation en marge des conventions dramatiques. Elle apparait dès alors comme l'expression singulière d'une création nomadique, à travers laquelle le dramaturge cherche à rendre le drame perméable à la modernité littéraire.

\section{Nomadiser le drame : une évolution vers le postdramatique?}

Le nomadisme propre à cette écriture est-il l'expression d'un théâtre postdramatique ? Selon Hans-Thies Lehmann, celui-ci se caractérise par une remise en cause de la primauté du "drame » au sens d'action et, plus généralement, du texte au profit d'une «juxtaposition et mise à niveau de tous les moyens confondus qui permettent au 
théâtre d'emprunter une pléthore de langages formels hétérogènes au-delà du drame " (2002 : 13). Cette nouvelle esthétique ne cesse en effet de tendre vers l'extra-théâtral, usant de formes intermédiales, voire interdisciplinaires, situées au croisement du théâtre, de la danse, de la performance, des nouvelles technologies et des arts plastiques et visuels. On pourrait citer, à titre d'exemple, les œuvres de Rodrigo García et Angélica Liddell, où l'écriture, mêlant les arts, est assumée par la mise en scène au sens large, c'est-à-dire par l'ensemble des médias constituant le spectacle.

Comme nous l'avons vu, l'œuvre de José Sanchis instaure, elle aussi, une ouverture sur des pratiques créatives et des champs de connaissance extérieurs au drame pour expérimenter une poétique du discontinu qui rejoint la visée artistique de ce nouveau théâtre. On perçoit aussi dans son écriture d'autres signes postdramatiques, tels le recours répété à la métathéâtralité ou encore l'instauration d'un spectateur résolument actif. Toutefois, force est de constater que, si cette écriture prend ses distances avec une conception logocentrée de l'expression théâtrale, elle ne récuse pas pour autant le primat du texte. Et c'est peut-être là que réside la principale différence entre le nomadisme propre à cette œuvre et l'interdisciplinarité du théâtre postdramatique. Selon Hans-Thies Lehmann, «le discours du théâtre [c'est-à-dire la sémiologie de la représentation] occupe une position centrale» (2002: 20) dans cette nouvelle esthétique. Si une telle démarche créative n'implique pas nécessairement le refus du texte, le critique allemand insiste sur le fait que " [celui-ci n'apparaît plus que] comme élément, sphère et matériau de l'agencement scénique » (2002: 20). Il en résulte alors un théâtre performatif faisant essentiellement appel au sensoriel (sons, gestes et images).

La poétique de José Sanchis n'intègre pas ce déplacement du langage vers la sensorialité. L'ouverture sur l'extra-théâtral est mise au service d'une expérimentation proprement dramatique. Le dramaturge se montre d'ailleurs dubitatif quant à la portée artistique d'un théâtre se limitant à l'expérience sensorielle: aux antipodes d'une " apología de la sensorialidad que se diluye en cuanto cae el telón » (Boadella, Onetti, Sanchis \& Solano, 1999: 100), son écriture ne renonce pas à la production du sens par l'entremise de la dramaturgie et du langage. S'il fait le choix d'une esthétique du discontinu, c'est précisément pour privilégier la polysémie, c'est-à-dire une production du sens alternative à une représentation figée de la réalité.

Envisagée de la sorte, cette pratique nomadique suppose une résistance vis-à-vis du drame conventionnel mais aussi du théâtre postdramatique. Comme le souligne Gilles Deleuze, «historiquement les nomades ne sont pas ceux qui bougent [mais], au contraire, ce sont ceux qui ne bougent pas, et qui se mettent à nomadiser pour rester à la même place en échappant aux codes" (2002: 362). La poétique de José Sanchis fonctionne sur un mode semblable: elle ne quitte pas la forme dramatique mais la nomadise pour échapper au Système théâtral bourgeois. Ainsi donc, loin de ressortir au théâtre postdramatique, elle participe de cette pulsion rhapsodique qui, selon Jean-Pierre Sarrazac, est à l'œuvre dans certaines écritures dramatiques contemporaines :

Pulsion permanente de renouvellement, d'émancipation par rapport à la norme - drame-dans-la-vie. Pulsion d'irrégularité, qui se manifeste de façon plus forte, voire impérative [...] à l'époque actuelle. Pulsion vers l'hétérogène, vers l'assemblage d'éléments disparates qui concerne aussi bien les grands modes d'expression tels que le dramatique, l'épique, le lyrique, l'argumentatif que la combinaison du comique, du tragique, du pathétique. Ou encore que l'inclusion de l'oralité dans l'écriture. (2007 : 15) 
Jean-Pierre Sarrazac prend ainsi ses distances avec ceux qui vaticinent la mort du drame ou son dépassement par d'autres esthétiques théâtrales, préférant mettre au jour la puissance nomadique d'écritures qui émancipent le drame en défaisant la complétude du «bel animal» aristotélicien. Cette nomadisation génère une transformation de la forme dramatique en la poussant vers des hybridations avec d'autres arts et d'autres disciplines qui la rendent plus souple et plus libre. L'adaptation proposée par José Sanchis du texte joycien apparaît dès lors comme une manifestation de cette hybridation formelle : le monologue intérieur ouvre la voie à une construction inédite de la pièce qui ne repose plus sur l'intrigue mais privilégie, au contraire, l'effacement de l'action théâtrale pour instaurer une théâtralité discursive.

En investissant la frontière comme espace de création, son écriture participe donc de cette pulsion rhapsodique, en cela qu'elle cherche à sortir du drame par le drame, non pas pour traverser la frontière mais pour la repousser et faire ainsi advenir une expérimentation en marge du Système théâtral bourgeois. En somme, la nomadisation du drame survient lorsqu'il y a résistance à l'unité dramatique et désir de déconstruire et réinventer la forme de manière permanente.

L'œuvre de José Sanchis, on le voit, participe de ce tournant spatial qui traverse les arts et le discours critique depuis une trentaine d'années. Elle instaure une rupture avec une approche généalogique qui envisage le théâtre en fonction de son histoire, au profit d'une réflexion transversale et ouverte sur d'autres arts et d'autres disciplines. Le dramaturge constate à cet égard que «el teatro ignora, o finge ignorar, lo que está ocurriendo en otros campos de la literatura o del arte, por no hablar de la ciencia » (2012:11). Pour y remédier, il en explore les frontières de sorte que le recours à l'extra-théâtral est mis au service d'une déconstruction de la théâtralité conventionnelle. L'adaptation apparaît dès lors comme la pratique nomadique par excellence car elle permet de confronter le Système théâtral bourgeois à des œuvres porteuses d'un renouveau formel pour ménager, selon les termes du dramaturge, "un espacio de cuestionamiento permanente de los estereotipos que, desde la teatralidad vigente, esclerotizan tanto la escritura dramática como la puesta en escena » (1998 : 162). L'intérêt qu'il porte à l'écriture de James Joyce participe de ce mouvement général : le travail d'adaptation lui permet d'importer dans le champ théâtral une poétique du discontinu qui déroge aux principes d'unité et de cohérence propres au «bel animal » aristotélicien. On voit ainsi comment, à travers ce nomadisme créatif, des arts et des disciplines extérieurs pénètrent dans le drame pour le transformer. Cette poétique de la frontière nous convie, en somme, à une réinvention constante. Et c'est peut-être là que réside toute la singularité de José Sanchis Sinisterra : avoir fait de l'écriture une puissance nomade, émancipatrice et créatrice en cela qu'elle libère inlassablement le drame de ses propres servitudes.

\section{BIBLIOGRAPHIE}

BAtLle Carles (1999), « Prólogo », J. Sanchis Sinisterra (dir.), El lector por horas, Barcelona :

Editorial Pro, 7-20.

ILCEA, $41 \mid 2020$ 
BOADELla Albert, ONETTI Antonio, SANCHIS SINISTERRA José \& SOlANo Ana (1999), Sesiones de trabajo con los dramaturgos de hoy, Ciudad Real : Ñaque.

Cattan Nadine (2006), « Centre-Périphérie », C. Ghorra Gobbin (dir.), Dictionnaire des mondialisations, Paris : Armand-Colin, 93-100.

Collot Michel (2011), La Pensée-paysage : philosophie, arts, littérature, Arles : Actes Sud.

Collot Michel (2014), Pour une géographie littéraire, Paris : José Corti.

DeLEUZE Gilles (2002), L'île déserte et autres textes, Paris : Éditions de minuit.

DeLeUze Gilles \& GUATTARI Félix (2005), Qu'est-ce que la philosophie ?, Paris : Éditions de minuit.

Deleuze Gilles \& GuATtARI Félix (1980), Mille Plateaux, Paris : Éditions de minuit.

DUJARDIN Édouard (1931), Le monologue intérieur : son apparition, ses origines, sa place dans l'œuvre de James Joyce, Paris : Albert Messein.

FoucAult Michel (2004), « Des espaces autres », Empan, 54(2), 12-19.

GENETTE Gérard (1972), Figures III, Paris : Éditions du Seuil.

HELBO André (2006), Signes du spectacle. Des arts vivants aux médias, Bruxelles : Presses interuniversitaires européennes.

LEHMANN Hans-Thies (2002), Le théâtre postdramatique, Paris : L'Arche.

MAYORGA Juan (2002), « Romper el horizonte: la misión de José Sanchis Sinisterra », J. Sanchis Sinisterra (dir.), La escena sin límites (fragmentos de un discurso teatral), Ciudad Real : Ñaque, 25-28. MeNETRIER Jacques (1972), Origines de l'Occident (nomades et sédentaires), Paris : Weber.

RYAN Marie-Laure (2003), « Narrative Cartography: Toward a Visual Narratology », T. Kindt \& H.H. Müller (dir.), What is Narratology? Questions and Answers Regarding the Status of a Theory, Berlin, New York : Walter de Gruyter, 333-364.

Ríos CARRATALÁ Juan Antonio (2009), « ¿No puede el teatro llevar a cabo la aventura hacia la abstracción que otras artes figurativas emprendieron a lo largo del siglo xx? (Entrevista a José Sanchis Sinisterra) », J. Sanchis Sinisterra (dir.), La máquina de abrazar, Madrid : Huerga y Fierro editores, 13-32.

SANCHIS SinISTERRA José (1991a), Perdida en los Apalaches (juguete cuántico), Madrid : Ministerio de Cultura.

SANCHIS SINISTERRA José (1991b), Ñaque o de piojos y actores et ¡Ay, Carmela!, Madrid : Cátedra. SANCHIS SINISTERRA José (1996), Tres dramaturgias. La noche de Molly Bloom, Bartleby el escribiente, Carta de la Maga a Bebé Rocamadour, Madrid : Fundamentos.

SANCHIS SINISTERRA José (1997), Pervertimento y otros gestos para nada, Madrid : Visor.

SANCHIS SINISTERRA José (1998), « Primera aproximación a un posible diccionario ideológico del teatro ", S. Fondevila (dir.), José Sanchis Sinisterra, l'espai fronterer, Barcelone : Institut del Teatre, 160-166.

SANCHIS SINISTERRA José (2002), La escena sin límites (fragmentos de un discurso teatral), Ciudad Real : Ñaque.

SANCHIS SINISTERRA José (2010), Próspero sueña Julieta (o viceversa) et Sangre lunar, Madrid :

Fundamentos. 
SANCHIS SINISTERRA José (2012), Narraturgia: dramaturgia de textos narrativos, México : Paso de Gato.

SARRAZAC Jean-Pierre (2007), « La reprise (réponse au postdramatique) », Études théâtrales (38-39), 7-18.

WeSTPHAL Bertrand (2007), La géocritique : réel, fiction, espace, Paris : Éditions de Minuit.

WHITE Kenneth (1987), L'esprit nomade, Paris : Le livre de poche.

\section{NOTES}

1. C'est en ces termes que José Sanchis définit ce système : "Erige, como paradigma de la teatralidad, la concreción en tácita complicidad de una figuración de la vida humana que se presenta como emanación del Discurso del autor, organizada según un sistema de convenciones y códigos preestablecidos tradicionalmente, preparada y ensayada con antelación y con pretensiones de invariabilidad; su naturaleza figurativa se basa en el pacto ficcional de una mayor o menor identidad entre los actores y los personajes, cuyas acciones configuran la imagen de un microcosmos coherente que se presenta como analogon de la realidad» (2002: 221). Présenté comme tel, ce système apparaît comme un avatar du théâtre aristotélicien. Le dramaturge insiste sur sa nature éminemment idéologique, reprenant ainsi à son compte la critique brechtienne à l'égard de la Poétique d'Aristote.

2. L'influence de José Sanchis sur Sergi Belbel, Lluïsa Cunillé ou encore Carles Batlle, chefs de file du renouveau théâtral catalan dans les années 1990, est indiscutable et nombreux sont les auteurs qui la reconnaissent comme telle. Carles Batlle souligne ainsi que «Cataluña tiene una gran deuda con José Sanchis Sinisterra. [...] Ha sido el maestro indiscutible de buena parte de los autores desde el año noventa para acá. Sus famosos laboratorios -o seminarios-de dramaturgia, lo mismo que sus clases en el Institut del Teatre de Barcelona, han sido la generosa cepa donde han brotado algunas de las mejores ramas de la dramaturgia catalana contemporánea » (1999: 7-8).

3. Alain Reynaud développe, à partir des années 1980, l'opposition centre/périphérie dans le domaine de la géographie pour la définir comme système territorial sans pour autant lui donner un sens purement géométrique. Nadine Cattan a en effet montré que, dans l'approche de Reynaud, «le centre n'est pas au milieu d'un espace, ni la périphérie reléguée aux marges. Le centre se caractérise par la concentration, en un lieu, d'une certaine masse de population, de fonctions économiques, d'activités de production et de services, de richesses. Il est doté d'une capacité d'innovation et de créativité. Bénéficiant d'une grande accessibilité, il est un lieu très attractif. Par les polarisations qu'il engendre, il est un puissant moteur de l'intégration territoriale. Toutefois, la dissymétrie des échanges est à la base de cette intégration. Avec des niveaux de vie moins élevés, la périphérie, souvent enclavée et isolée, envoie des flux migratoires massifs vers le centre. Elle est dépendante, subordonnée et se décline toujours en négatif par rapport au centre. Sa faiblesse principale tient de l'absence d'autonomie en matière décisionnelle. À l'instar des couples infernaux, centre et périphérie sont indissociables. Ils ne prennent sens que dans la relation dialectique qui les unit » (2006:45).

4. José Sanchis définit le concept du translucide comme « aquello que no es ni transparente ni opaco, sino que permite entrever, adivinar, intuir, pero deja también mucha ambigüedad $e$ indeterminación » (Juan Antonio Ríos Carratalá, 2009 : 28). 


\section{RÉSUMÉS}

La visée théâtrale de José Sanchis Sinisterra est indissociable d'un mouvement déterritorialisant qui déborde le drame conventionnel et l'entraine vers ses dehors, là où il est possible d'instaurer des zones de voisinage avec d'autres domaines de création et d'autres champs de connaissance. Nous voulons envisager cette poétique comme l'expression d'un nomadisme intellectuel afin d'en déterminer les implications esthétiques dans le domaine de la création théâtrale : faut-il y voir l'expression singulière d'un théâtre résolument interartistique ou une revendication du texte théâtral d'autant plus assumée qu'elle en assure la déconstruction?

El proyecto teatral de José Sanchis Sinisterra responde a una desterritorialización que desborda el drama convencional y explora sus fronteras para instaurar zonas de vecindad con otras formas de creación y otros ámbitos de conocimiento. Nos proponemos analizar dicha poética como la expresión de un nomadismo intelectual para determinar cuáles son sus implicaciones en la creación teatral: ¿debemos considerarla como la expresión singular de un teatro decididamente interartístico o como una reivindicación del texto teatral con el fin de desconstruirlo?

José Sanchis Sinisterra's theatrical project responds to a deterritorialization which goes beyond conventional drama and explores its borders in order to establish neighborhood zones with other forms of creation and other fields of knowledge. We want to consider this poetics as the expression of an intellectual nomadism in order to determine its aesthetic implications in the field of theatrical creation: is it the singular expression of an interartistic theater or a claim of the theatrical text to deconstruct this one?

\section{INDEX}

Mots-clés : nomadisme, déterritorialisation, adaptation théâtrale, extra-théâtralité, théâtre postdramatique

Keywords : nomadism, deterritorialization, theatrical adaptation, extra-theatricality, postdramatic theatre

Palabras claves : nomadismo, desterritorialización, adaptación teatral, extrateatralidad, teatro posdramático

\section{AUTEUR}

\section{LAURENT GALLARDO}

ILCEA4 (Université Grenoble Alpes) 